\title{
Oralité et colonialité au prisme de la diglossie littéraire réunionnaise
}

\author{
Philippe Glâtre ${ }^{11}$ \\ ${ }^{1}$ Université Sorbonne Nouvelle Paris 3 - UMR 7107 LaCiTO (CNRS, Paris 3, Inalco), Paris, France.
}

\begin{abstract}
Résumé. Si l'histoire des rapports entre écriture et oralité met en perspective des agencements permanents, la Modernité occidentale a donné une place prépondérante à la littératie. Dans la France hexagonale des XIX et $\mathrm{XX}^{\mathfrak{e}}$ siècles, la tradition orale et les langues régionales ont alors connu une dépréciation au profit de la langue nationale écrite. En même temps que des pratiques oratoires telles que la rhétorique et la poésie orale étaient exclues de l'enseignement, les écrits en langue minoritaire, particulièrement s'ils étaient à visée littéraire, étaient assignés à la littératie vernaculaire et la culture populaire. Le retour contemporain de l'oralité, particulièrement visible dans l'émergence du slam, se heurte donc au statut prépondérant donné à l'écrit et à la langue hégémonique. Dans les sociétés coloniales françaises, le système scripturaire a également été conçu comme un instrument de civilisation, mais réservé à une élite favorable au système colonial. La scolarisation a donc été basée sur la francisation, restreignant ainsi l'éducation des subalternes à une littératie rudimentaire. Ce processus de minorisation a eu des conséquences sociolinguistiques importantes dans les territoires français d'Outre-mer, qui conjuguent diglossie et colonialité. A l'Île de La Réunion, où le créole est la langue première de la majorité de la population, le rapport à l'écrit et à la langue dominante a ainsi longtemps participé d'une exclusion des textes créolophones de la littérarité. Il faudra attendre la fin du $\mathrm{XX}^{\mathfrak{e}}$ siècle pour que soit remise en cause une littératie monolingue et que la tradition orale créole retrouve sa propre historicité linguistique et culturelle.
\end{abstract}

\begin{abstract}
Orality and coloniality through the prism of the Reunionese literary diglossy. By developing an overview of the permanent historical arrangements that define the relation between writing and orality, we seek to demonstrate that Western Modernity assigned to literacy a predominant status. In France, during the XIX ${ }^{\text {th }}$ and the $\mathrm{XX}^{\text {th }}$ centuries, oral tradition and regional languages have been depreciated in favor of the written national language. As oratory practices like rhetoric and oral poetry were excluded from schooling, literary writings in minorized languages were assigned to vernacular literacy and folklor. The contemporary come-back of orality, particularly noticeable in the emergence of slam poetry, meets with the predominant status given to written and hegemonic language. In the French colonial societies, the scriptural system was also conceived as an instrument of civilization, but reserved for an elite favorable to the colonial system. Learning was therefore based on francization, thus restricting the education of subalterns to rudimentary literacy. This minorization had important sociolinguistic consequences on French Overseas Territories,
\end{abstract}

1 philippe.glatre@sorbonne-nouvelle.fr 
that combine diglossy and coloniality. On Reunion Island, whereas Creole is the first language of the majority of the population, the relationship with the written word and the dominant language thus participated for a long time in the exclusion of Creole-speaking texts from literarity. It is only at the end of the $\mathrm{XX}^{\text {th }}$ century that monolingual literacy has been questioned and that Creole oral tradition recovered its own linguistic and cultural historicity. 
Depuis son invention au paléolithique et sa systématisation autour de -3100 dans l'Ouest de l'Asie, l'expansion de l'écriture a souvent été associée au développement de sociétés complexes et urbanisées (Goody, 1991, 76). D'abord utilisée pour des fonctions administratives et économiques, elle est rapidement devenue un instrument au service d'États centralisés ayant besoin d'administrer de larges territoires. Cette vision évolutionniste pourrait alors ne laisser voir qu'un lent développement de la littératie qui assoirait son pouvoir sur l'oralité au fur et à mesure de sa diffusion. Pourtant, quand apparaît l'écriture dans une société de tradition orale, littératie et oralité entretiennent des relations complexes, dans lesquelles se jouent d' "incessants processus d'imposition et d'appropriation, d'acceptation et d'opposition provenant de toutes parts " (Rockwell, 2010, 98). Chaque époque, chaque société, indexicalise en fait le rapport écrit-oral en fonction de son organisation (Duranti, 2009).

Dans l'antiquité, les sociétés indo-européennes ont longtemps donné la primauté à l'oralité (Bodelot, 2017, 17-18). A l'inverse, la Chine a très tôt instauré une domination de l'écrit (Hagège, 1985, 108-109), tout comme les premiers chrétiens qui pour se distinguer de la culture grecque et du prestige donné à la parole publique, se sont détournés de cette tradition orale en christianisant la culture écrite (Fournet, 2019). La latinité antique a pour sa part privilégié l'oralité, la voix y étant omniprésente, même dans le processus de production de textes écrits (Bodelot, op. cit., 20-21). A la fin de l'Empire romain d'Occident jusqu'aux débuts de la Chrétienté latine, il existe une certaine homogénéité entre langue écrite et parlée, qui va se fissurer à partir du VII ${ }^{\mathrm{è}}$ siècle et surtout avec la réforme carolingienne et le concile de Tours en 813 , qui entérine le fait que le latin ne soit plus réservé qu'à l'écrit (Ibid, 24-25). Il devient alors la langue savante, en opposition aux langues romanes, une diglossie (Prudent, 1981) que les Serments de Strasbourg viendront réagencer en 842 , en inventant des écritures aux langues romanes. Mais toujours est-il que l'usage de l'écrit reste le fait d'une élite, religieuse et intellectuelle pour le latin, juridique et politique pour les langues vulgaires, et que les milieux populaires devront attendre de nombreux siècles pour s'approprier progressivement l'écrit, dans leurs propres langues (Balibar, 1985, 412-413 ; Noiriel, 2018, 62-63).

Cette situation de littératie dominante (Barton \& Hamilton, 2010, 58-59) a en effet perduré au cours du Moyen-Âge, même si l'imprimerie et les écrits vernaculaires, notamment épistolaires, témoignent d'un accès à l'écrit des milieux populaires (Chartier, 2001, 787-789). Mais c'est paradoxalement à l'Époque moderne, quand l'alphabétisation des milieux populaires tend à se généraliser, que la culture occidentale radicalise la domination de l'écrit sur l'oral, particulièrement au cours des XIX è ${ }^{\grave{e}}$ XX ${ }^{\grave{e}}$ siècles, parenthèse lors de laquelle la littératie a été conçue comme un instrument de subalternisation de la tradition orale (Legoy \& Vaillant, 2017, 11-12).

Alors que l'on assiste aujourd'hui à un "retour de l'oralité » (Fraisse, 2013, 339), il est intéressant de s'arrêter sur l'époque contemporaine, notamment à travers le prisme des littératures orales, qui illustrent les enjeux de pouvoir entre cultures écrites et orales. Ce phénomène prenant une acuité particulière en situation coloniale, là où la Modernité occidentale déploie son épistémologie avec le plus de force (Sousa Santos, 2011). A cet égard, les espaces francophones postcoloniaux sont des terrains très éclairants, du fait de la place particulière que prend la littératie dans la société française. L'île de La Réunion, en particulier, de par sa situation diglossique, est traversée par les rapports de pouvoir entre littératie et oralité. Une analyse sociolinguistique de sa littérature contemporaine permet alors de mieux comprendre comment la situation coloniale indexicalise la littérarité de sa tradition orale. 


\section{1 - L’oralité minorisée par la modernité}

Si les recherches récentes portant sur les rapports entretenus entre écriture et oralité aboutissent à la conception d'un continuum plutôt qu'à une dichotomie entre ces dernières (Fraenkel \& Mbodj, 2010), donc à une relativisation de l'importance de la littératie dominante, il reste néanmoins que la modernité conçoit l'oralité comme un ensemble de pratiques subalternes. Au cours du XIX è siècle, c'est donc par le prisme du folklore que les chercheurs commencent à se pencher sur les pratiques orales. Or ce terme, conçu par l'Anglais William Thoms, renvoie à la transmission orale, mais aussi à des productions considérées comme un "savoir du peuple » (Bornand \& Leguy, 2013, 111). Logiquement, cette approche s'est donc surtout orientée à l'époque vers le recueil de textes, fonctionnant comme la trace de pratiques culturelles populaires amenées à disparaître.

Dans cette perspective évolutionniste, l'oralité est conçue comme un atavisme ayant pour finalité l'homéostase (Goody, 1979, 53 ; 2007, 44), dans une société pensée anhistorique, là où le scripturaire fait fonction de mythe pour les sociétés occidentales (De Certeau, 2010, 198-199). Selon Michel de Certeau la littératie est pensée, depuis les Lumières, comme participant d'un progrès, à travers la capacité qu'a l'écrit de modeler celui qui le pratique. Le système scripturaire est donc considéré comme un vecteur de civilisation des classes populaires (Ibid, 241-242). L'histoire de Jean Le Liseur, exhumée par Jean-Marie Privat, est à cet égard représentative de l'époque. Dans la France du XIX ${ }^{\mathrm{e}}$ siècle, notamment au sortir de la défaite de 1870, la République produit un effort systématique d'alphabétisation afin d'acculturer à l'écrit des populations rurales encore majoritairement rétives à l'univers de l'écrit. Une " révolution culturelle » qui visait in fine à "substituer définitivement la culture écrite et éclairée à la culture orale et obscurantiste » (Privat, 2007, 5-6).

Cette minorisation est d'ailleurs visible dans la dichotomie instituée entre écrit et oral, à laquelle on pourrait superposer "populaire/savant» (Zumthor, 1982, 121), et que l'on retrouve dans ce que Roger Chartier a appelé « révolution de la lecture silencieuse ». Dans un processus qui court du Moyen-Âge au XIX ${ }^{\grave{e}}$ siècle, la notion d'illettrisme a glissé progressivement vers l'incapacité à lire "seulement avec les yeux et en silence " (Chartier, 2009, 101). Au XIX , la discrimination entre lettrés et illettrés est donc fondée non pas sur l'accès à l'écrit, mais sur un effacement de l'oralité dans la pratique de la lecture.

A la même époque, un autre phénomène est significatif de la dépréciation de la tradition orale : la suppression progressive dans l'enseignement en France de la rhétorique. Inventée dans la Sicile grecque autour de -465 (Reboul, 2013, 14), cette pratique a perduré durant plus de deux millénaires, sans changer fondamentalement dans sa forme. Mais dans une France qui s'inventait dans un néo-classicisme gréco-romain, elle a paradoxalement disparu, jusqu'au mot lui-même, des manuels scolaires (Ibid, 11). Parallèlement, un autre genre de discours se voit minorisé dans les écoles françaises : la poésie orale. Depuis la plus haute antiquité, la poésie était surtout une pratique qui avait affaire avec le chant et la déclamation, mais peu avec l'écrit (Legoy \& Vaillant, op. cit., 12-13). C'est là encore au cours du XIX ${ }^{\mathrm{e}}$ siècle qu'a émergé une sacralisation du livre, au détriment d'autres formes poétiques sans « vocation bibliophilique » $(\mathrm{Ibid}, 14)$. L'École de la III ${ }^{\mathrm{e}}$ République, dans sa volonté de construire un " culte national des grands genres », a alors érigé la poésie au seul rang de littérature écrite et "substitué à la réalité vivante de la poésie une illusion figée et artificielle " (Ibid, 18). A partir des années 1880, la pratique de la poésie en classe se limite à une poésie scolaire, basée sur la récitation et la diction, et c'est principalement à l'instituteur que revient le loisir de sa pratique orale, et de sa maîtrise (Fabre, 1997, 40). 
On retrouve à travers ces exemples la distinction bakhtinienne entre "genres de discours premiers », perçus comme simples, et "seconds», plus complexes, souvent artistiques, scientifiques ou politiques, et liés à la pratique écrite (Bakhtine, 1984, 295). Cette hiérarchie des genres indique là encore une minorisation de l'énonciation orale à l'époque moderne, dont l'idéologie linguistique n'avait pu laisser place à aucune nomenclature des genres du discours oral au moment des travaux de Bakhtine (Ibid, 295). La poésie orale se voit alors assignée au " genre familier », en opposition à la poésie écrite officielle, rendant alors absente une vision populaire du monde liée à la tradition orale (Ibid, 337).

Bien sûr, cette sous-estimation de la valeur esthétique des pratiques orales ne les a pas fait disparaître pour autant, la poésie orale constituant encore aujourd'hui une part importante des écrits littéraires, en particulier dans les sociétés minorisées. Mais c'est au prix d'une folklorisation de ces pratiques. Chikako Mori, travaillant sur la littératie en milieu populaire, montre que dans la société française de la fin du $\mathrm{XX}^{\mathrm{e}}$ siècle, qui donne une place prépondérante à l'écrit, il était difficilement concevable que les jeunes de banlieue puissent avoir des pratiques culturelles littéraires. A ses débuts, le rap a donc été assigné à une oralité spontanée, renvoyant au sauvage, là où la littératie se présente comme une pratique raisonnable (Mori, 2012, 75). Au-delà du rapport hiérarchique entre écrit et oral, l'épistémologie moderne construit en fait une dichotomie entre culturel et social, et le fait de priver les littératures orales de leur dimension artistique empêche la tradition orale de s'élever au rang de civilisée (Carinos \& Hammou, 2017, 273). Dans une perspective évolutionniste, l'expression verbale renvoie alors à la primitivité, ce qui fut longtemps le cas pour les arts de la parole des peuples colonisés. Il est à cet égard significatif que Wole Soyinka, qui deviendra prix Nobel de littérature en 1986, se soit vu refuser dans les années 1970 un poste au sein du département de lettres de l'Université de Cambridge, mais fut par contre accepté dans celui d'anthropologie sociale (Okpewho, 1986, 20).

Dans une société de tradition orale, "l'effraction de l'écriture " (Hagège, op. cit., 121) met donc en péril la transmission de pratiques que la modernité subalternise, au risque de déshistoriciser certains savoirs. La perspective réductionniste instituée par l'appropriation de la littérature entraîne donc l'émergence de pratiques orales cherchant à perpétuer une tradition populaire exclue par des élites lettrées. Un phénomène particulièrement prégnant au sein des sociétés minorisées. François Paré, qui travaille sur l'émergence du slam au Canada, considère que ce dernier a été inventé par la communauté portoricaine de NewYork et est ensuite rapidement devenu un espace privilégié de défense culturelle et linguistique pour des minorités diasporiques et autochtones d'Amérique de Nord (Paré, 2015, 92). Selon lui, sa diffusion est donc liée au souci de transmission de cultures orales mises à mal par le colonialisme. A cet égard, il est intéressant de noter que le slam a rapidement franchi la frontière des États-Unis vers le Canada, mais qu'il a été diversement accueilli dans ce pays. Le Canada anglophone s'est en effet approprié très vite ce genre, mais le Québec a d'abord eu quelques réticences à sa pratique, pour deux raisons. Il a tout d'abord été perçu comme trop anglo-saxon, donc comme possible danger pour la culture francophone (Fraisse, op. cit., 342). Il est aussi probablement apparu comme moins nécessaire à une société québécoise moins «désoralisée » que la société française de la fin du siècle dernier (De Granpré, 1986, 229). Ce phénomène est particulièrement intéressant, dans la mesure où il met en perspective les interactions sociolinguistiques entre langue, oralité et écriture. Car si la société québécoise a d'abord maintenu ses distances avec cet art oratoire, c'est qu'il existait déjà une tradition de la lecture poétique (Fraisse, op. cit., 349). C'est peut-être aussi parce que l'accès aux livres francophones y était moins aisé (Paré, op. cit., 96), le français restant à l'échelle du pays une langue minoritaire. Ce maintien d'une tradition orale permettait donc de résister à la langue et la culture anglo-saxonnes, et il 
faudra attendre que le slam prenne racine à Paris, centralité de la francophonie, pour que les Québécois le trouvent plus présentable et se l'approprient (Fraisse, op. cit., 342). On voit ici que la dimension culturelle peut parfois prendre le pas sur des questions purement linguistiques (Derive, 2017, 287).

Cette émergence du slam canadien ne fut pas sans créer des tensions avec les acteurs plus anciens des lectures poétiques, qui l'ont d'abord perçu comme une pratique aux canons insuffisamment littéraires (Fraisse, op. cit., 356-367). Un texte du poète québécois JeanPhilippe Tremblay, en réaction aux performances des slameurs québécois, est significatif des réagencements qu'entraîne l'irruption de genres de discours secondaires dans les pratiques dominantes. En intitulant sa performance $A$ un jeune powète (Ibid), il s'adresse à des slameurs pratiquant un art oratoire populaire quand lui se place plutôt du côté de Rainer Maria Rilke et de la littérature écrite. Mais c'est justement parce que les soirées de lecture poétique étaient devenues une pratique élitiste que le slam a pris une place permettant à des genres de discours plus familiers de faire "pénétrer dans la littérature des couches de langue qui, jusque-là, se trouvaient frappées d'interdit de langage " (Bakhtine, op. cit., 337).

\section{2 - Francophonie et littératie dominante}

En France, le slam s'ancre dans une société qui avait ôté à la "parole vive » (Chartier, $2009,96)$ son caractère littéraire et tente de redonner ses lettres de noblesse à une tradition orale. Il s'invente à travers un retour aux sources de l'oralité, dans une filiation avec la poésie antique (Vorger, 2011, 151) et tente donc d'opérer un continuum entre tradition et modernité, venant pallier notamment à la disparition de la rhétorique. Car l'entreprise de civilisation par le scripturaire, dans la France des $\mathrm{XIX}^{\mathfrak{e}}$ et $\mathrm{XX}^{\mathfrak{e}}$ siècles, s'est accompagnée d'une standardisation de la langue écrite, consubstantielle de la construction des Étatsnations (Canut, 2001, 446). Les langues périphériques ont alors été reléguées au statut de langues non-écrites et par là-même exclues de la culture savante. Là encore c'est tout un pan de la société qui se voit minorisé par un double processus diglossique (Fabre, op . cit., 7-8). La langue minoritaire est distinguée de la langue officielle, devenue dominante, et elle est empêchée d'accès à la littératie dominante, car trop éloignée de l'écrit standard. Ce projet républicain de dépréciation des langues régionales a amené les militants occitanistes, notamment, à forger le concept de "colonialisme intérieur » pour signifier les similitudes existant entre la minorisation des traditions orales de l'Hexagone et celle des Outre-mers (Lagarde, 2012, 44-45).

L'expansion coloniale a en effet été particulièrement violente dans son intention de déshistoricisation des langues de tradition orale. Forgés par le "primat de l'écriture » (Hagège, op. cit., 90-91), les premiers missionnaires à se pencher sur les langues des colonisés, bien que souvent dépourvus de compétence linguistique dans ces langues étrangères, les ont décrites comme fluctuantes et pauvres. Ils accréditaient ainsi la thèse encore très palpable dans les travaux de Goody qui veut que les langues sans tradition écrite ne permettent que difficilement de transmettre une histoire et d'accéder à la complexité. S'inspirant de la métaphore de la page blanche chez De Certeau (Op. cit., 199-200), Kasereka Kavwehiri considère que la colonisation occidentale, pour réduire au silence les peuples colonisés, a usé de l'écrit comme d'une arme de déshistoricisation : "Rien ne doit rester en dehors de la main-mise du pouvoir colonial qui produit/écrit l'histoire ; l'histoire de l'expansion et de la domination européenne ; l'histoire, en somme, de la domination de l'écriture sur l'oralité, de la civilisation sur la barbarie, de l'ordre sur le désordre. Écrire, c'est prendre possession, maîtriser l'ambivalent, " homogénéiser " pour introduire dans un ordre historique déterminé » (Kavwehiri, 2004, 797). Phénomène qu'illustrent les travaux 
de Ranajit Guha sur les révoltes indiennes à l'époque de l'Empire britannique. Dans les écrits relatant ces événements, c'est le discours du colonisateur qui s'impose progressivement, donnant aux productions écrites la capacité de construire a posteriori un événement allant dans le sens du discours colonial (Guha, 2017, 28-29).

Dans les sociétés coloniales françaises, c'est l'articulation de la scolarisation et de la langue française qui fonctionne comme un instrument privilégié de civilisation. Dans l'optique de diffuser la langue nationale, et ce dès 1826 en ce qui concerne l'Afrique de l'Ouest, un arrêté stipule que les " jeunes négresses » sénégalaises devront être instruites uniquement en français (Goheinex, 2012, 86). Toutefois, si la généralisation de cet apprentissage fait consensus au sein des autorités coloniales, la question se pose jusqu'aux indépendances de savoir quel degré d'instruction est nécessaire. Car la francisation s'accompagne d'un "pragmatisme pédagogique », adapté à des intelligences "limitées » (Ibid, 84), qui restreint l'apprentissage de la langue à des rudiments fonctionnels en prenant soin d'éviter aux enfants colonisés un accès à des niveaux conceptuels trop élevés. Seule une minorité, forgée par l'épistémè coloniale, peut avoir accès à des études plus poussées, afin de fournir aux institutions des médiateurs dociles et fidèles au système colonial. C'est donc une inquiétude politique qui pousse à former une élite locale aux subtilités de la langue et à restreindre les masses à un apprentissage rudimentaire. Les autorités veillent ainsi scrupuleusement au contenu des bibliothèques, pour éviter d'ouvrir l'esprit des enfants colonisés qui pourraient, via des lectures dangereuses, remettre en cause les bienfaits de la colonisation (Ibid, 90-91). La francisation dans les colonies visait en définitive à produire une société hiérarchisée compatible avec des besoins économiques (Lucas, 2002 ; 2005, 23) et basée sur l'accès à la langue nationale. Ce faisant, elle instituait une double subalternité, fondée sur une diglossie toujours à l'œuvre dans ces territoires et l'exclusion des langues régionales du système éducatif.

Fort d'une idéologie langagière fondée par le monolinguisme (Canut, 2005), l'enseignement dans les Outre-mers français a imposé la langue française comme médium d'enseignement et d'entrée dans la littératie, faisant abstraction des situations sociolinguistiques locales, fondées sur le plurilinguisme des territoires et le multilinguisme de la population. Jusqu'à très récemment, l'utilisation dans les établissements scolaires des langues autochtones, pidgins et créoles, faisait l'objet de punition. Si ce n'est plus le cas aujourd'hui, on peut tout de même voir cette logique opérer dans les dichotomies spatiales instituées dans les écoles. Les langues locales sont beaucoup plus présentes dans les cours d'école que dans les classes, au sein desquelles le plurilinguisme est toléré, mais au prix d'une séparation et d'une hiérarchisation des langues. En Guyane notamment, le français et les langues locales sont incarnés par des enseignants différents, à des horaires différents et assignés à des espaces segmentés : le fond de la classe pour les langues régionales, le tableau pour le français (Léglise, 2017, 257). Malgré la décolonisation des territoires ultramarins français, l'Éducation nationale s'appuie toujours sur une vision du bilinguisme conçu comme " soustractif », la langue première des enfants n'étant bien souvent acceptée qu'à l'oral (Ibid, 255), comme vecteur d'assimilation de la langue officielle, seul accès possible à la littératie. Encore une fois, nous voyons que les langues de tradition orale subissent une double subalternisation : cantonnées à une oralité diglossique et empêchées d'écrit.

\section{3 - Littérarité et colonialité à La Réunion}

L'Île de la Réunion présente des singularités culturelles et linguistiques qui aident à préciser les liens entretenus par l'oralité et la littératie. A la différence des autres territoires ultramarins, la colonisation s'est fondée sur un peuplement allogène sans population autochtone 
(Vergès, 2008, 231). En l'absence de passé précolonial, elle ne peut être qualifiée de société orale au sens de Jack Goody et Ian Watt, l'écrit et l'oral y ayant coexisté dès le début du peuplement (Magdelaine-Andrianjafitrimo, 2008, 196), mais plutôt de «littératie restreinte » (Goody, 2007, 20-22) dès lors que l'écrit est limité à des groupes sociaux et des individus spécifiques.

Le créole réunionnais est une langue résultant des hybridations entre les différentes populations successivement arrivées sur l'île. La première attestation du "parler créole réunionnais » remonterait au début du XVIII ${ }^{\mathfrak{e}}$ siècle (Georger, 2011, 62), le créole s'inventant dès les premières décennies de la colonisation comme système de communication orale (Véronique, 2013). Peu après, au milieu du même siècle, des locuteurs bilingues et lettrés produisent les premiers écrits en créole, dont la trace a été retrouvée en 2004 dans les archives de la Congrégation Lazariste à Paris (Hélias, 2014, 55). C'était surtout le fait, à l'époque, de missionnaires qui avaient le souci d'évangéliser la population dans le «parler des Noirs » (Robert Chaudenson, cité par Hélias, op. cit., 57). Les premiers textes littéraires en créole remontent pour leur part à la fin du XVIII ${ }^{\grave{e}}$. Ce sont d'abord des contes et chansons populaires français traduits en créole par des écrivains issus de la haute société (Georger, op. cit., 311). Plus tard apparaissent les fables, les premières connues étant les Fables Créoles dédiées aux dames de l'Ile Bourbon, publiées par Louis Héry en 1828 (Hélias, op. cit., 60). Elles consistent, de prime abord, en la traduction de fables de La Fontaine, mais à y regarder de plus près, elles sont aussi adaptées au contexte local et permettent à son auteur, par le ressort de l'ironie, de prendre une distance avec la langue française, ce qui fait que ce texte est aujourd'hui considéré comme la pierre fondatrice de la littérature en créole réunionnais (Georger, op. cit., 312).

$\mathrm{Au}$ cours du $\mathrm{XIX}^{\mathrm{e}}$ siècle et jusque 1950 cependant, la littérature créole produira finalement peu d'écrits (Hélias, op. cit., 20) et restera cantonnée à des genres folklorisés (Lauret, 2017, 124-125). Car pour être reconnue en tant que telle, une œuvre littéraire doit aussi comprendre une "méta-littérature ", à savoir " un appareil critique de gloses, de commentaires, d'analyses » (Prudent, cité par Hélias, op. cit., 243). Or la situation coloniale réunionnaise a donné une visibilité aux œuvres francophones "hantées par la créolisation » (Magdelaine-Andrianjafitrimo, op. cit., 191), particulièrement au roman colonial (Marimoutou, 2014), instituant alors une "diglossie littéraire » (Peter Hawkins, cité par Hélias, op. cit., 409). En tant que langue de tradition orale, il était attendu que le créole réunionnais ne produise que des textes simples, dénués d'un caractère littéraire réservé aux oeuvres francophones. Jusque la fin du $\mathrm{XX}^{\mathrm{e}}$ siècle, les anthologies littéraires réunionnaises n'ont d'ailleurs laissé que peu de place à la littérature d'expression créole (Magdelaine-Andrianjafitrimo, op. cit., p.189), et il faudra finalement attendre deux siècles pour que les premières fables retrouvent une légitimité et soient canonisées (Hélias, op. cit., 189).

Passée de colonie à département français en 1946, La Réunion a connu un essor important du système éducatif, alors que la majorité de la population avait encore une instruction très sommaire (Lucas, 2005, 19). Cet effort républicain reposant alors sur une francisation, La Réunion a perpétué les principes de littérarité valables en France hexagonale : ne pouvait être littéraire qu'un texte écrit et de langue française. De fait, les débuts de la départementalisation n'ont vu que peu d'œuvres éditées, en français comme en créole (Hélias, op. cit., 219) ; mais au début des années 1960 et surtout durant les années 1970 émerge un courant d'intellectuels qui remettent en cause les canons coloniaux. Coïncidant avec le moment de la décolonisation de l'Empire français, ce sont alors différentes visions de la "créolité du texte » qui se dégagent à La Réunion (Georger, op. cit., 312). 
Le premier courant, connu sous le nom de créolie, tente de valoriser la culture créole réunionnaise par l'usage de la langue nationale. Pour ces écrivains, la légitimité littéraire passe donc majoritairement par l'écriture en français, pour inscrire la littérature créole dans le champ de la "francophonie réunionnaise " (Ibid, 313). Le discours de Gilbert Aubry, acteur de ce courant et aujourd'hui évêque de La Réunion, est significatif de cette démarche : "la Créolie s'exprimera tant en créole qu'en français et le français reste la langue privilégiée à étudier et à maîtriser, parce qu'au plan de l'expression elle est nettement supérieure au créole et vous situe au niveau de la communication et de la recherche internationale " (Aubry, cité par Georger, 313). Principalement poètes, ces auteurs ont donc cherché à s'approprier la langue du maître, dans une volonté d'assimilation que Gérard Noiriel conçoit comme la principale forme de résistance des peuples colonisés ( $O p$. cit., 166). Mais en restant conditionnés par la situation diglossique locale, ils ont malgré eux participé à la marginalisation de la langue et des écrits créolophones.

Le second courant, qualifié de créoliste, revendique le droit à une littérature créole orale, mais surtout écrite (Georger, op. cit., 316). Il s'agissait de s'affranchir du " monolinguisme triomphant» (Magdelaine-Andrianjafitrimo, op. cit., 191) pour faire entendre une vision du monde autre, en faisant reconnaître le créole réunionnais comme langue à part entière, mais aussi comme langue d'écriture littéraire. C'est tout d'abord dans les milieux étudiants réunionnais de France hexagonale, au début des années 1960, que sont produits les premiers poèmes créolophones. A La Réunion, c'est surtout au cours des années 1970 que ce courant a pris un essor important (Georger, op. cit., 314-316) et participé à l'émergence d'une "littérature de résistance " (Magdelaine-Andrianjafitrimo, op. cit., 192). C'est un tournant important dans la société réunionnaise car pour la première fois des auteurs se positionnaient en rupture avec l'ordre colonial. En ayant recours à la langue vernaculaire, ils relativisaient la diglossie et faisaient entrer la poésie créole dans le champ de la littérature. En toile de fond il s'est agi, pour ces artistes militants, d'équiper la langue créole pour rendre possible l'exploration de tous les genres littéraires (Georger, op. cit., 318). Alors que pour les tenants de la créolie, c'est l'écriture dans une langue seconde qui permettait l'émancipation, pour les créolistes c'est la langue régionale qui a été conçue comme une arme (Ibid, 316).

Si ces courants avaient pour préoccupation de sortir la littérature réunionnaise des canons européens, leurs tiraillements indiquent surtout une volonté d'émancipation par l'écrit. C'est donc autour de la question du livre et des langues subalternes que s'articulent leurs positionnements. Mais pour d'autres auteurs, la liminalité est celle du rapport écritoral. Refusant le livre comme critère de littérarité, ils préfèrent donner à entendre leurs textes in situ, lors de performances qui prennent souvent la forme du kabar fonnkèr, genre poétique créole réunionnais issu de la tradition est-africaine et malgache (Wells, 2018 ; Pongérard, 2009 ; Fuma, 2004). Dans leur optique, c'est donc par l'oralité que doivent circuler les poèmes, en opposition au format individuel du livre, associé à la culture occidentale. A travers cette démarche, il s'agit aussi de s'adresser à un large public, ce que ne permet pas la publication écrite. Car si le discours déplorant la diminution de la lecture de poésie est récurrent dans les élites littéraires françaises depuis deux siècles, force est de constater que les livres de poésie n'ont jamais touché qu'un lectorat très restreint (Legoy \& Vaillant, op. cit., 14). C'est probablement ce qui explique que depuis les années 1970 le champ littéraire créolophone s'oriente vers la diversification des supports : l'enregistrement audio de poèmes, la chanson, le théâtre ou encore le conte (Magdelaine-Andrianjafitrimo, op. cit., 195). Cette évolution témoignant là aussi de l'effraction de genres de discours jusqu'alors considérés comme paralittéraires. 
Bien que les différents courants de la littérature réunionnaise contemporaine indiquent des positionnements sociolinguistiques différents, ils témoignent d'une tentative de relativiser la distinction entre langue dominante et vernaculaire, ainsi qu'entre littérature orale et écrite. En sapant les bases narratives coloniales (Bertho, 2018, 202), ils participent d'un travail de réhistoricisation de la culture créole, qui passe par la réappropriation d'un 《savoir-pouvoir » (Amselle, 2001, 77) confisqué par l'Occident. Reste à comprendre dans quelle mesure cette libération de la parole subalterne, en tant qu'elle subit malgré tout un processus de codification qui peut la rendre élitiste, peut être accessible au plus grand nombre. Un travail d'indexicalisation de l'énonciation (Duranti et al, 2012 ; Scott, 2019 ; Spivak, 2009 ; Butler, 2008) pourrait alors permettre de mieux situer le droit à la parole publique dans une société réunionnaise qui oscille toujours entre émancipation et situation coloniale.

Mots-clés : Langue créole, littérature orale, minorisation, slam

Keywords: Creole langage, oral literature, minorization, slam poetry 


\section{Références bibliographiques}

Amselle, J.-L. (2001). Branchements. Anthropologie de l'universalité des cultures. Paris : Flammarion.

Bakhtine, M. (1984). Esthétique de la création verbale. Paris : Gallimard.

Balibar, R. (1985). L'institution du français. Essai sur le colinguisme des Carolingiens à la République. Paris : PUF.

Barton, D., \& Hamilton, M. (2010). La littératie : Une pratique sociale. Langage et société, 133, 45-62.

Bertho, E. (2018). Fabrique d'un canon: Entre archives coloniales, fait littéraire et culture populaire (Sarraounia, Niger). In S. Bornand \& J. Derive (Éd.), Les canons du discours et la langue : Parler juste (197-222). Paris : Karthala.

Bodelot, C. (2017). Latin parlé / latin écrit : De la symbiose à la diglossie. Langages, 208, 17-28.

Bornand, S., \& Leguy, C. (2013). Anthropologie des pratiques langagières. Paris : Armand Colin.

Butler, J. (2008). Le pouvoir des mots: Discours de haine et politique du performatif. Paris : Éditions Amsterdam.

Canut, C. (2001). À la frontière des langues. Cahiers d'études africaines, 163-164, 443-464.

Canut, C. (2005). Mélanges, pidgins, créoles. Africultures, (62), 56-63.

Carinos, E., \& Hammou, K. (2017). Approche du rap en français comme forme poétique. In S. Hirschi, C. Legoy, A. Saemmer, A. Vaillant, \& S. Linarès (Éd.), La poésie délivrée (227-240). Nanterre : Presses universitaires de Paris Nanterre.

Chartier, R. (2001). Culture écrite et littérature à l'âge moderne. Annales. Histoire, Sciences Sociales, 56e année, (4), 783-802.

Chartier, R. (2009). Les usages de l'écrit du Moyen Âge aux Temps modernes. Entretien avec Roger Chartier. Propos recueillis par Étienne Anheim et Pierre Chastang. Médiévales [En ligne], 56, 93-114. https://doi.org/10.4000/medievales.5564

De Certeau, M. (2010). L'invention du quotidien. T.1 Arts de faire (Nouvelle éd). Paris : Gallimard.

De Grandpré, C. (1986). La poésie comme parole. Voix et Images, 11(2), 228-240.

Derive, J. (2018). Les propriétés canoniques de quelques genres du discours en pays mandingue et leurs incidences linguistiques (phonologie, morphologie, syntaxe, lexique). In S. Bornand \& J. Derive (Éd.), Les canons du discours et la langue: Parler juste, 267-288. Paris : Karthala.

Duranti, A. (2009). L'oralité avec impertinence. L'Homme, Oralité et écriture, 189, 23-47.

Duranti, A., Ochs, E., \& Schieffelin, B. (2012). The Handbook of Language Socialization. Malden/Oxford: Wiley-Blackwell.

Fabre, D. (1997). Introduction. Seize terrains d'écriture. In D. Fabre (Éd.), Par écrit : Ethnologie des écritures quotidiennes, 1-56. Paris : Éditions de la Maison des Sciences de l'Homme.

Fournet, J.-L. (2019, février 6). Le calame et la croix : La christianisation de l'écrit et le sort de la culture classique dans l'Antiquité tardive. Cours au collège de France. https://www.college-defrance.fr/site/jean-luc-fournet/course-2019-02-06-11h00.htm

Fraenkel, B., \& Mbodj-Pouye, A. (2010). Introduction. Les New Literacy studies, jalons historiques et perspectives actuelles. Langage et société, 133, 7-24.

Fraisse, P. (2013). Langue, identité et oralité dans la poésie du Québec (1970-2010). Des nuits de la poésie au slam: Parcours d'un engagement pour une culture québécoise. (Thèse de Doctorat en linguistique, Université de Cergy Pontoise). https://tel.archives-ouvertes.fr/tel-00957943

Fuma, S. (2004). Aux origines ethno-historiques du maloya réunionnais traditionnel ou «le maloya réunionnais, expression d'une interculturalité indiaocéanique ». In Y.-S. Live \& J.-F. Hamon (Éd.), Diversité et spécificités des musiques traditionnelles de l'océan Indien (207-218). Paris : L'Harmattan.

Georger, F. (2011). Créole et français à La Réunion: Une cohabitation complexe (Thèse de linguistique, Université de la Réunion). https://tel.archives-ouvertes.fr/tel-01279041

Goheneix, A. (2012). Stratification linguistique et ségrégation politique dans l'Empire français: L'exemple de L'AOF (1903-1945). Glottopol, revue de sociolinguistique en ligne, Linguistiques et colonialismes, 20, 82-103.

Goody, J. (1979). La raison graphique : La domestication de la pensée sauvage. Paris : Éditions de Minuit.

Goody, J. (1991). Alphabets et écriture. Réseaux, 9(48), 69-93. 
Goody, J. (2007). Pouvoirs et savoirs de l'écrit. Paris : La Dispute.

Guha, R. (2017) . La prose de la contre-insurrection. In Subaltern Studies : une anthologie, (2171). Éditions de l'Asymétrie : Toulouse.

Hagège, C. (2002). L'homme de paroles. Contribution linguistique aux sciences humaines. Paris : Fayard.

Hélias, F. (2014). La poésie réunionnaise et mauricienne d'expression créole: Histoire et formes. Ille-sur-Têt : Éditions K’A.

Kavwahirehi, K. (2004). La littérature orale comme production coloniale. Notes sur quelques enjeux postcoloniaux. Cahiers d'études africaines, 44(176), 793-813.

Lagarde, C. (2012). Le «colonialisme intérieur»: D'une manière de dire la domination à l'émergence d'une « sociolinguistique périphérique » occitane. Glottopol, revue de sociolinguistique en ligne, Linguistiques et colonialismes, 20, 38-54.

Lauret, F. (2017). L'humour créole réunionnais: Dynamique linguistique et culturelle (19632011) (Thèse de Doctorat en Linguistique, Université de la Réunion). https://tel.archivesouvertes.fr/tel-01623602

Léglise, I. (2017). Multilinguisme et hétérogénéité des pratiques langagières. Nouveaux chantiers et enjeux du Global South. Langage et société, 160-161, 251-266.

Legoy, C., \& Vaillant, A. (2017). Introduction générale. In S. Hirschi, A. Saemmer, S. Linarès, C. Legoy, \& A. Vaillant (Éd.), La poésie délivrée (11-24). Nanterre: Presses universitaires de Paris Nanterre.

Lucas, R. (2002). Esclavage et scolarisation à l'île Bourbon : le projet pédagogique des Frères des écoles chrétiennes. In Edmond Maestri (Éd.), Esclavage et abolitions dans l'océan Indien (17231860). Actes du colloque de Saint-Denis de La Réunion du 4-8 décembre 1998 (49-61). Paris/SaintDenis : L'Harmattan/Université de la Réunion.

Lucas, R. (2005). Le développement de la scolarisation... Un cas d'école? In A. Si Moussa (Éd.), L'école à La Réunion: Approches plurielles (11-49). Paris : Karthala.

Magdelaine-Andrianjafitrimo, V. (2008). Littératures de La Réunion, littératures plurielles. Hommes et Migrations, 1275(1), 188-197.

Marimoutou, J.-C. C. (2014). Représentations et écritures aux frontières. Le roman colonial mauricien et réunionnais. Les Cahiers Naturalistes, 88, 81-100.

Mori, C. (2012). L'archipel invisible. L'écriture dans les “cultures de banlieue". Hommes \& migrations, 1297, 68-76.

Noiriel, G. (2018). Une histoire populaire de la France: De la guerre de Cent Ans à nos jours. Marseille : Agone.

Okpewho, I. (1986). The Study of African Oral Literature. Présence africaine, 3(139), 20-40.

Paré, F. (2015). Esthétique du Slam et de la poésie orale dans la région frontalière de GatineauOttawa. Voix et Images, 40(2), 89-103.

Pongérard, P. (2009). Ethnographie du "Servis Kaf", un rituel sacré réunionnais, d'origine africaine. In Laurent Médéa (ed.), Kaf: étude pluridisciplinaire (175-189). Sainte-Clotilde [Réunion] : Zarlor éditions.

Privat, J.-M. (2007). Jean Bête et Jean Alphabète. Cahiers de littérature orale [En ligne], 62, 1-14. https://doi.org/10.4000/clo. 1214

Prudent, L.-F. (1981). Diglossie et interlecte. Langages, 15(61), 13-38.

Reboul, O. (2013). Introduction à la rhétorique: Théorie et pratique. Paris : PUF.

Rockwell, E. (2010). L'appropriation de l'écriture dans deux villages nahua du centre du Mexique. Langage et société, 133, 83-99.

Santos, B. de S. (2011). Épistémologies du Sud. Études rurales, 187, 21-49.

Scott, J. C. (2019). La domination et les arts de la résistance: fragments du discours subalterne. Paris : Éditions Amsterdam.

Spivak, G. C. (2009). Les subalternes peuvent-elles parler?. Paris : Éditions Amsterdam.

Vergès, F. (2008). La Réunion: Un modèle de vivre ensemble. Hommes \& Migrations, L'interculturalité en débat, Hors-Série, 20-29.

Véronique, G. D. (2013). Émergence des langues créoles et rapports de domination dans les situations créolophones. In Situ. Revue des patrimoines [En ligne], 20, 1-18. https://doi.org/10.4000/insitu.10209

Vorger, C. (2011). Poétique du slam: De la scène à l'école. Néologie, néostyles et créativité lexicale (Thèse de Doctorat en Sciences du langage, didactique et linguistique, Université de Grenoble). http://www.theses.fr/2011GRENL017 
Wells, H. (2018). Moving Words, Managing Freedom: The Performance of Authority in Malagasy Slam Poetry ( $\mathrm{PhD}$ thesis in Anthropology, UC Berkeley). https://escholarship.org/uc/item/36f7m3s3

Zumthor, P. (1982). Le rythme dans la poésie orale. Langue française, Le rythme et le discours, sous la direction de Henri Meschonnic, 56, 114-127. 